Robotica: (2019) volume 37, pp. 2119-2131. (C) Cambridge University Press 2019. This is an Open Access article, distributed under the terms of the Creative Commons Attribution-NonCommercial-NoDerivatives licence

(http://creativecommons.org/licenses/by-nc-nd/4.0/), which permits non-commercial re-use, distribution, and reproduction in any medium, provided the original work is unaltered and is properly cited. The written permission of Cambridge University Press must be obtained for commercial re-use or in order to create a derivative work. doi:10.1017/S0263574719000626

\title{
Effectiveness of a Robot-Mediated Strategy While Counteracting Multidirectional Slippages
}

\author{
F. Aprigliano $\dagger, \perp \odot$, V. Monaco $\dagger, \ddagger, \perp^{*}$, P. Tropea $\llbracket$, \\ D. Martelli§, N. Vitiello $\nmid \ddagger$ and S. Micera $\dagger, \|$
}

$\dagger$ The BioRobotics Institute, ScuolaSuperioreSant’Anna, Pisa, Italy. E-mails: federica.aprigliano@ santannapisa.it; nicola.vitiello@santannapisa.it

$\ddagger$ IRCCS Fondazione Don Carlo Gnocchi, Milan, Italy. E-mail: vito.monaco@santannapisa.it

ФDepartment of Neurorehabilitation Sciences, Casa CuraPoliclinico, Milan, Italy.

E-mail: p.tropea@ccppdezza@it

$\S$ Department of Mechanical Engineering, Columbia University, New York, NY, USA.

E-mail:dm3042@columbia.edu

\|Bertarelli Foundation Chair in Translational NeuroEngineering, Center for Neuroprosthetics and Institute of Bioengineering, School of Engineering, Ecole Polytechnique Federale de Lausanne, Lausanne, Switzerland.E-mail: silvestro.micera@santannapisa.it

(Accepted April 2, 2019. First published online: June 14, 2019)

\section{SUMMARY}

This study investigates the effectiveness of a robot-mediated strategy aimed at promoting balance recovery after multidirectional slippages. Six older adults were asked to manage anteroposterior and mediolateral slippages while donning an active pelvis orthosis (APO). The APO was set up either to assist volunteers during balance loss or to be transparent. The margin of stability, in sagittal and frontal planes, was the main metric to assess the effectiveness of balance recovery. Results showed that the assistive strategy is effective at promoting balance recovery in the sagittal plane, for both perturbing paradigms; however, it is not effective at controlling stability in the frontal plane.

KEYWORDS: Balance control; Wearable robot; Multidirectional slippages; Margin of stability; Elderly people.

\section{Introduction}

Falls are universally acknowledged to be one of the most disabling conditions affecting the health and quality of life of the population especially for most industrialized countries. Several epidemiological studies agree on the evidence that about one-third of people older than 65 years fall once per year. ${ }^{1}$ Falls are a cause of a substantial rate of morbidities and mortalities, as well as one of the major contributors to functional deterioration, immobility, premature nursing home placement, and hospitalization. ${ }^{2}$ In addition, fear of falling and related loss of confidence lead fallers to reduce their social interactions and physical activities, thus further impairing muscle strength, agility, balance control, and, even more, increasing the risk of a fall. ${ }^{2-5}$ Counteracting the risk of a fall is hence of primary importance to allow the growing elderly population to remain safe while maintaining autonomy during daily activities, and to improve their overall quality of life.

\footnotetext{
$\perp$ Equally contributed and alphabetic order.

*Corresponding author. E-mail: vito.monaco@santannapisa.it
} 
Many authors have developed and tested different tools combining machine learning approaches and suitable motor tasks to predict the risk of a fall. ${ }^{6}$ Others have demonstrated the effectiveness of prevention programs, based on training, ${ }^{7,8}$ behavior, and environmental modifications, ${ }^{9}$ to reduce the probability of accidents. Overall, all these preventing strategies, whose effectiveness is well documented, are usually designed to improve balance control and/or to reduce the risk of balance loss for specific cohorts of persons. However, they are not useful to counteract a lack of balance due to unexpected hazards met during daily activities (e.g., slippery surfaces and carpets) which can suddenly escalate to a fall.

To address this last issue, we have recently tested the effectiveness of a proactive closed-loop strategy combining a predictive algorithm, able to detect signs of lack of balance, and a wearable robotic exoskeleton, supplying an assistive strategy. ${ }^{10}$ We tested this approach in both healthy elderly people and transfemoral amputees while managing unexpected slippages delivered by a split-belt treadmill along the anterior-posterior (AP) direction. Although promising, the validity of the results is limited by several issues, including the adopted perturbation paradigm. Indeed, it involved only forward slippages in spite of the evidence that a lack of balance can be due to several causes, such as multidirectional slippages, tripping, and stumbling. In more detail, from the biomechanical viewpoint, a fall is an intrinsically complex motor event whose variability depends on both the psycho-physical status of the actor and the perturbation itself (e.g., its type and direction). In addition, it is well known that the stabilization of human movements in the frontal plane can be more challenging than in the sagittal plane. ${ }^{11,12}$ Due to this anisotropy, different control strategies are expected to be required to counteract the lack of balance after multidirectional perturbations.

According to this evidence, the outcome of our previous study ${ }^{10}$ cannot be generalized to different experimental conditions. Therefore, in the present study we tested the hypothesis that the robotmediated strategy to counteract the balance loss described in ref. [10] is still effective while subjects manage unexpected multidirectional slippages.

\section{Materials and Methods}

\subsection{Participants, experimental setup, and protocol}

Six healthy older adults (six males, $68.7 \pm 5.2$ years old, $76.9 \pm 7.9 \mathrm{~kg}, 1.76 \pm 0.1 \mathrm{~m}$ ) were enrolled for this study. The exclusion criteria were: age greater than 80 years, falls documented in the last 6 months, relevant comorbidities (e.g., hemiplegia, degenerative nervous system diseases, chronic heart failure, chronic obstructive pulmonary disease, and severe sensory deficits), poor cognitive skills (Mini-Mental State Examination score $<24$ ), inability to walk safely on a treadmill, and severe anxiety or depression. Participants were informed of the purpose of the study and signed informed consent forms before the experimental sessions began. All research procedures were in accordance with the Declaration of Helsinki and were approved by the Institutional Ethics Committee of the Don Gnocchi Foundation (Florence, Italy), where the experiments took place.

The experimental protocol was the same adopted in ref. [10] and mainly consisted in asking participants to manage unexpected multidirectional slippages delivered while they were steadily walking at their preferred speed $(0.89 \pm 0.12 \mathrm{~m} / \mathrm{s})$. Perturbations were provided by SENLY (Fig. 1a; [13]), a custom-made mechatronic platform consisting of a two split-belt treadmill whose belts can be independently moved both longitudinally and transversally (i.e., the right belt can be moved in all clockwise directions from north to south, and the left belt can be moved in all directions in the remaining hemiplane). In this study, perturbations were delivered on the right side and consisted of a sudden forward (AP) or lateral (mediolateral; ML) movement of the right belt triggered by the heel strike of the right foot. Velocity profiles for both perturbations are reported in refs. [12,14]. Subjects donned a safety harness attached to an overhead track to prevent possible impacts with the ground, and started the experimental tests after 3 min of acclimation while walking on SENLY at their preferred speed.

During the experimental sessions, the subjects wore an active pelvis orthosis (APO; [15]), which is a powered wearable robot designed to assist hip flexion-extension (Fig. 1a). The control strategy driving the APO can switch between two working modalities, viz., zero-torque and assistive modes (Z- and A-modes, respectively). The former (Z-mode, i.e., transparent mode; see [16]) is designed to be extremely compliant to the user's intention, allowing subjects to freely perform movements 
(a)

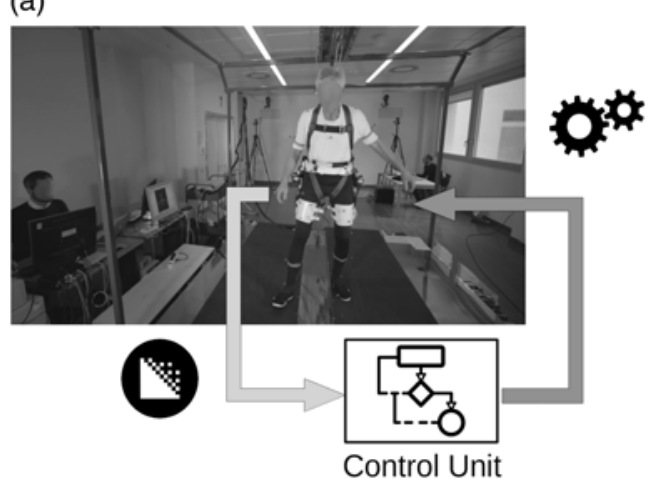

(b)

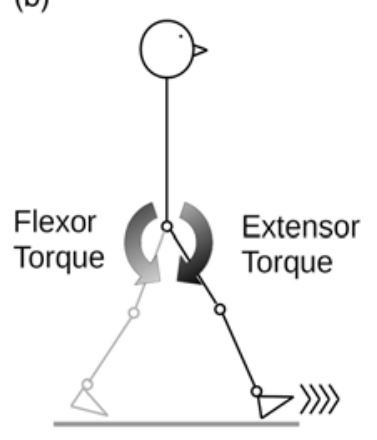

Fig. 1. Overview of the robot-mediated assistive strategy. (a) The mechatronic platform delivers an unexpected slippage while the subject is wearing the active pelvis orthosis (APO) and steadily walking on the treadmill. The balance loss is detected by the algorithm running in the APO control unit and parsing the hip joint angles (light gray line). Then a counteracting strategy, mediated by the APO, is delivered to promote balance recovery (dark gray line). (b) The panel shows a subject managing a slippage on the perturbed limb (black), while the APO supplies extensor and flexor torques at the hip joints of the perturbed and unperturbed (gray) limbs, respectively.

without being obstructed by the device. The latter (A-mode, i.e., assistive mode) is always transparent until balance loss is detected. In this case, the robot supplies assistive torques at both hip joints to promote stability recovery (Fig. 1b).

The assistive strategy (A-mode) combines a detection algorithm ${ }^{17}$ with a suitable set of torque patterns supplied at the hip joints. Specifically, the detection algorithm runs in the control unit of the APO, comparing the actual kinematics of the robot (hip joint angles in the sagittal plane) with those predicted by a pool of adaptive oscillators (AOs). In steady conditions, the input (i.e., current kinematics) and output (i.e., predicted kinematics) of the AOs are likely to be comparable, with zero time lag. Thus the amplitude of their difference (i.e., the error) is pretty small. Due to a sudden perturbation, the periodic features of the gait patterns are lost and the AOs are no longer able to accurately predict the current kinematics, thus the error sharply increases over a certain threshold. The algorithm thus detects the loss of balance and enables the assistive strategy mediated by the APO. The assistive strategy consists of a couple of torque patterns aimed at simultaneously extending the perturbed limb (PL) and flexing the unperturbed limb (UL). The APO supplies assistive torques with a bell-shaped profile, whose amplitude is proportional to the gross (user and exoskeleton) weight, that is, $0.2 \mathrm{Nm} / \mathrm{kg}$, lasting $0.25 \mathrm{s.}{ }^{10}$ Noticeably, different assistive torque patterns provided by the APO were previously investigated. ${ }^{18}$ Nonetheless, the above-mentioned assistive strategy has been identified as the best combination of torque patterns to counteract the lack of balance after a slippage. Indeed, it slows down the slipping foot (i.e., the PL) and minimizes the downward movement of the $\mathrm{CoM}$, thanks to the opposite torque patterns between hips (Fig. 1b).

Experimental sessions comprised two repetitions for each perturbation (i.e., AP and ML) and each robot-working modality (i.e., Z- and A-modes). Six additional fake trials, in which no perturbation was applied, were also included in the experimental protocol. Overall, the participants underwent 14 experimental trials (i.e., 2 repetitions $\times 2$ modes $\times 2$ perturbations and 6 fake trials). To obtain unbiased results: (i) the participants did not know whether they would have been perturbed or not; (ii) the participants did not know whether they would have been assisted by the APO or not; and (iii) the experimental conditions (i.e., AP or ML perturbations, Z- or A-modes, and true or fake trials) were supplied in random order.

The 3D trajectories of 34 spherical markers located on suitable body landmarks and 10 on the APO were recorded at $250 \mathrm{~Hz}$ using a 6-camera-based Vicon 512 Bonita 10 Motion Analysis System (Oxford, UK). Details of the markers' locations are described in ref. [10]. Kinematic records, belts movements, and APO-related kinematic/kinetic features were synchronized by means of a logic pulse generated by SENLY during the delivery of the perturbations.

\subsection{Data processing}

The coordinates of each marker were low-pass filtered with cut-off frequency at $10 \mathrm{~Hz}$ using a $4^{\text {th }}$ order zero-lag Butterworth filter, in order to remove the high-frequency-related noise. 
(a)

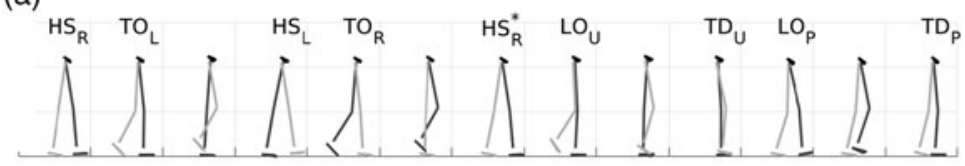

(b)

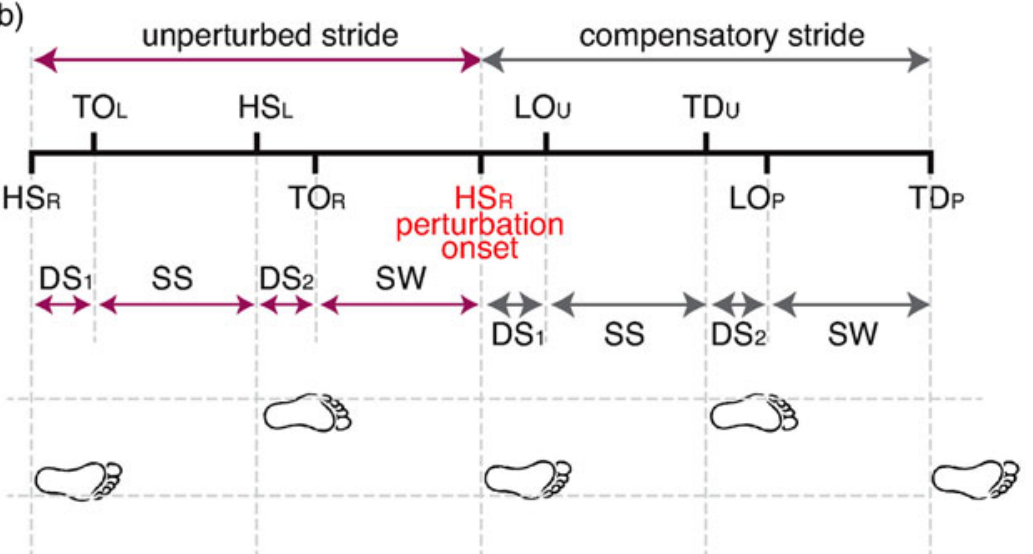

Fig. 2. Unperturbed and compensatory strides. (a) Stick diagram for two strides across the perturbation onset $\left(\mathrm{HS}_{\mathrm{R}}{ }^{*}\right)$. Dark and light gray lines refer to the perturbed (right) and the unperturbed (left) limbs, respectively. Labels highlight the time events. Heel strike and toe off are shown for the right $\left(\mathrm{HS}_{\mathrm{R}}\right.$ and $\left.\mathrm{TO}_{\mathrm{R}}\right)$ and left foot $\left(\mathrm{HS}_{\mathrm{L}}\right.$ and $\left.\mathrm{TO}_{\mathrm{L}}\right)$, before the perturbation onset. Touch down and lift off are shown for the right (i.e., perturbed; $\mathrm{TD}_{\mathrm{P}}$ and $\mathrm{LO}_{\mathrm{P}}$ ) and the left foot (unperturbed; $\mathrm{TD}_{\mathrm{U}}$ and $\mathrm{LO}_{\mathrm{U}}$ ) after the perturbation onset. (b) Phases of the gait cycle during unperturbed and perturbed strides. Temporal parameters are reported before and after the perturbation onset (purple and gray lines, respectively).

A full body biomechanical model was developed accounting for the following 15 segments: head/neck, chest, abdomen/pelvis, upper arms, forearms, hands, thighs, shanks, and feet. All joints were approximated as spherical and their centers were located in accordance with the literature. ${ }^{19,20}$ The time course of the right limb-related joint angles in the sagittal plane was estimated as the projection of the relative angle between the distal and proximal body segments in the plane of progression. ${ }^{21}$ In addition, we similarly estimated the time course of the right hip joint angles in the frontal plane.

The range of motion (RoM) was computed for the hip, knee, and ankle joint angles in the sagittal plane $\left(\mathrm{RoM}_{\mathrm{Hs}}, \mathrm{RoM}_{\mathrm{K}}\right.$, and $\left.\mathrm{RoM}_{\mathrm{A}}\right)$, and hip joint angle in the frontal plane $\left(\mathrm{RoM}_{\mathrm{Hf}}\right)$, as the difference between the maximum and the minimum values during both the unperturbed and compensatory strides across the onset of the perturbation and related to the right limb. The whole body center of mass (CoM) was approximated by using the sacral marker, in accordance with ref. [22].

Gait events across the perturbation onset (i.e., heel strike/touch down and toe off/lift off of both feet; see Fig. 2a) were identified by visual inspection of both the feet kinematics and the ground reaction forces after being properly filtered to remove drift and high frequency artefacts (a pass-band filter with cut offs at 0.15 and $10 \mathrm{~Hz}$; refs. [23,24]).

For each subject and each trial, the data were subdivided into two subsets: data recorded before (PRE) and after (POST) the onset of the perturbation, as shown in Fig. 2. The former referred to the last right unperturbed stride, starting with the heel strike before the perturbation onset and ending at the heel strike triggering the perturbation. The latter referred to the compensatory stride; it started simultaneously with the end of the previous stride and ended with the following ipsilateral heel strike. After the onset of the perturbation, four consecutive time events were identified: the lift off of the unperturbed foot $\left(\mathrm{LO}_{\mathrm{U}}\right)$; the time frame immediately before (i.e., one frame, consisting of 1/250 s) the touchdown of the unperturbed foot $\left(\mathrm{TD}_{\mathrm{U}}\right)$; the lift off of the perturbed foot $\left(\mathrm{LO}_{\mathrm{P}}\right)$; and the time frame immediately before the touchdown of the perturbed foot $\left(\mathrm{TD}_{\mathrm{P}}\right)$. These four consecutive time events allow investigating the stability against balance loss, under the hypothesis that the balance recovery may require more than one step to be achieved.

Several temporal parameters were evaluated along the strides before and after the perturbation onset, as reported in Fig. 2b: the first double support phase $\left(\mathrm{DS}_{1}\right)$; the single support phase (SS) 

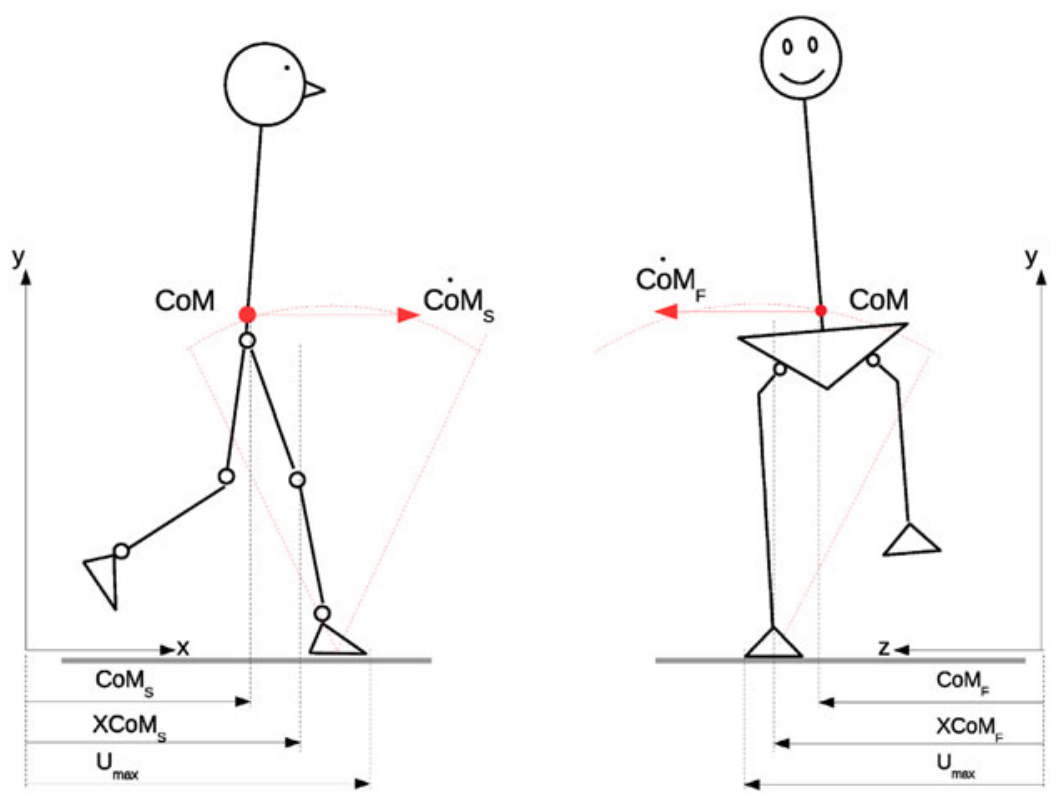

Fig. 3. Computing the margin of stability. The figure shows the components of center of mass $\left(\operatorname{CoM}_{S / F}\right)$ and related velocity $\left(C o \dot{M}_{S / F}\right)$, the $X C o M_{S / F}$ and the external boundary of the base of support $\left(U_{\max }\right)$, both in the sagittal and the frontal planes (left and right panels, respectively). Based on these metrics, it is possible to compute $M o S_{S / F}$.

related to the left limb; the second double support phase $\left(\mathrm{DS}_{2}\right)$; and the swing phase $(\mathrm{SW})$ of the right limb.

To investigate the effectiveness of the assistive strategy while promoting balance recovery, the margin of stability (MoS; Fig. 3) was computed in the sagittal and frontal planes, according to ref. [25], as

$$
M o S_{S / F}=U_{\max }-X \operatorname{CoM}_{S / F}
$$

where $U_{\max }$ is the external boundary of the base of support (i.e., fifth metatarsal head), and $X C o M_{S / F}$ is the extrapolated $C o M{ }^{26}$ This latter variable represents the projection of the $C o M$ under the hypothesis of pendular oscillation (i.e., accounting for the $\mathrm{CoM}$ velocity with respect to the belt) in the sagittal and frontal planes, and is computed as

$$
X \operatorname{CoM}_{S / F}=\operatorname{CoM}_{S / F}+\frac{\operatorname{Co} M_{S / F}}{\sqrt{\frac{g}{h}}}
$$

where $C o M_{S / F}$ and $C o \dot{M}_{S / F}$ are the components, in the sagittal and the frontal planes, of the position and velocity of the $C o M, h$ is the length of the equivalent pendulum, and $g$ is the gravitational acceleration. Both components of the $\mathrm{MoS}\left(\mathrm{MoS}_{\mathrm{S}}\right.$ and $\left.\mathrm{MoS}_{\mathrm{F}}\right)$ were quantified at time events before $\left(\mathrm{TO}_{\mathrm{L}}\right.$, $\mathrm{HS}_{\mathrm{L}}, \mathrm{TOR}$, and $\left.\mathrm{HS}_{\mathrm{R}}\right)$ and after $\left(\mathrm{LO}_{\mathrm{U}}, \mathrm{TD}_{\mathrm{U}}, \mathrm{LO}_{\mathrm{P}}\right.$, and $\left.\mathrm{TD}_{\mathrm{P}}\right)$ the perturbation.

\subsection{Statistical analysis}

All outcome variables (temporal and kinematic parameters, $\mathrm{MoS}_{\mathrm{S}}$ and $\mathrm{MoS}_{\mathrm{F}}$ ) were used as dependent measures.

A $t$-test for paired samples was used to investigate the effect of perturbation (before and after the onset of the perturbation). Then, considering only data after the onset of the perturbation (POST data), a two-way repeated measures ANOVA was performed to determine the main and simple interaction effects of the perturbation direction (two levels: AP and ML) and exoskeleton working mode (two levels: A- and Z-mode). If significant, the main effect of these analyses was followed up by pairwise comparisons with Bonferroni's correction. Statistical significance was set at $p<0.05$. 
Table I. Temporal parameters $\left(\mathrm{DS}_{1}, \mathrm{SS}, \mathrm{DS}_{2}\right.$, and $\mathrm{SW}$; mean \pm standard deviation, across subjects) before (PRE) and after (POST) the perturbation onset, during AP and ML slippages and while the APO was working in A- and Z-modes.

\begin{tabular}{lcccc}
\hline & & & \multicolumn{2}{c}{ POST } \\
\cline { 4 - 5 } Temporal parameter & Perturbation direction & PRE & A-mode & Z-mode \\
\hline $\mathrm{DS}_{1}[\mathrm{~s}]$ & $\mathrm{AP}$ & $0.13 \pm 0.02$ & $0.12 \pm 0.03$ & $0.12 \pm 0.03$ \\
& $\mathrm{ML}$ & $0.12 \pm 0.03$ & $0.10 \pm 0.03$ & $0.11 \pm 0.03$ \\
$\mathrm{SS}[\mathrm{s}]$ & $\mathrm{AP}$ & $0.38 \pm 0.04$ & $0.34 \pm 0.08$ & $0.35 \pm 0.06$ \\
& $\mathrm{ML}$ & $0.38 \pm 0.04$ & $0.36 \pm 0.05$ & $0.36 \pm 0.06$ \\
$\mathrm{DS}_{2}[\mathrm{~s}]$ & $\mathrm{AP}$ & $0.09 \pm 0.03$ & $0.05 \pm 0.03$ & $0.07 \pm 0.03$ \\
& $\mathrm{ML}$ & $0.09 \pm 0.02$ & $0.06 \pm 0.03$ & $0.05 \pm 0.02$ \\
$\mathrm{SW}[\mathrm{s}]$ & $\mathrm{AP}$ & $0.42 \pm 0.05$ & $0.26 \pm 0.08$ & $0.26 \pm 0.10$ \\
& $\mathrm{ML}$ & $0.41 \pm 0.05$ & $0.27 \pm 0.08$ & $0.28 \pm 0.07$ \\
\hline
\end{tabular}

Table II. Two-way repeated measures ANOVA on temporal parameters. $p$-values are reported for main effects of the perturbation direction $\left(p_{\text {dir }}\right)$ and the exoskeleton-working modality $\left(p_{\text {exos }}\right)$, and for their interaction $\left(p_{\mathrm{dir} * \mathrm{exos}}\right) . p<0.05$ are in bold.

\begin{tabular}{lccc}
\hline Temporal parameter & $\boldsymbol{p}_{\text {dir }}$ & $\boldsymbol{p}_{\text {exos }}$ & $\boldsymbol{p}_{\text {dir*exos }}$ \\
\hline $\mathrm{DS}_{1}$ & 0.097 & 0.802 & 0.470 \\
$\mathrm{SS}$ & 0.293 & 0.565 & 0.299 \\
$\mathrm{DS}_{2}$ & 0.827 & 0.231 & $\mathbf{0 . 0 2 7}$ \\
$\mathrm{SW}$ & 0.755 & 0.959 & 0.478 \\
\hline
\end{tabular}

\section{Results}

\subsection{Temporal parameters}

Table I shows the temporal parameters observed before (PRE) and after (POST) the perturbation onset, due to the AP and the ML slippages, while the APO was working in A- and Z-modes. A significant effect of the perturbation was observed on all temporal parameters, which decreased during the compensatory stride ( $t$-test; $\mathrm{DS}_{1}: p=0.047$; SS: $p=0.019$; $\mathrm{DS}_{2}$ and SW: $p<0.0001$ ).

Considering the POST data, results of the two-way ANOVA (Table II) revealed that temporal parameters were not significantly affected by the perturbation direction (AP vs. ML) or the APO working modality (A vs. Z). A significant interaction between the two factors was observed for the second double support phase: during the A-mode, $\mathrm{DS}_{2}$ was higher for ML than for AP slippages; during the Z-mode, $\mathrm{DS}_{2}$ was lower for ML than for AP slippages.

\subsection{Lower limb kinematic}

The lower limb angular excursions are depicted in Fig. 4.

RoM in the sagittal $\left(\mathrm{RoM}_{\mathrm{Hs}}, \mathrm{RoM}_{\mathrm{K}}\right.$, and $\left.\mathrm{RoM}_{\mathrm{A}}\right)$ and the frontal $\left(\mathrm{RoM}_{\mathrm{Hf}}\right)$ planes of the PL are reported in Table III. After the perturbation (i.e., PRE vs. POST), RoM $\mathrm{Hf}_{\mathrm{Hf}}$ significantly increased while $\mathrm{RoM}_{\mathrm{K}}$ and $\mathrm{RoM}_{\mathrm{A}}$ significantly decreased ( $t$-test, $p<0.0001$ in all cases). RoM $\mathrm{Hs}_{\mathrm{Hs}}$ was not affected by the perturbation ( $t$-test, $p=0.143$ ).

Concerning the POST data, the assistive strategy significantly modified the hip RoM in the sagittal plane (Table IV), showing lower values during the A-mode than during the Z-mode, as reported in Table III.

\subsection{Effectiveness of the assistive strategy}

3.3.1. Dynamic stability in the sagittal plane. The values of the sagittal component of the MoS are reported in Table V. Our analysis revealed that the perturbation significantly modified the MoSS at HSL/TDU, TOR/LOP, and HSR/TDP ( $t$-test, $p<0.0001$ in all cases). There were no differences when the MoSS was calculated at TOL/LOU ( $t$-test, $p=0.194)$. 
Table III. Range of motion (RoM) of joint angles (mean \pm standard deviation, across subjects) before (PRE) and after (POST) the perturbation onset, during AP and ML slippages and while the APO was working in A- and Z-modes.

\begin{tabular}{lcccc}
\hline & & & \multicolumn{2}{c}{ POST } \\
\cline { 4 - 5 } RoM & Perturbation direction & PRE & A-mode & Z-mode \\
\hline Hip $_{S}[\mathrm{deg}]$ & AP & $35.6 \pm 8.2$ & $27.2 \pm 5.9$ & $32.8 \pm 7.1$ \\
& ML & $34.8 \pm 6.4$ & $32.7 \pm 11.9$ & $37.4 \pm 9.2$ \\
Hip $_{F}[\mathrm{deg}]$ & AP & $7.4 \pm 1.7$ & $14.4 \pm 5.4$ & $15.4 \pm 7$ \\
& ML & $8.2 \pm 1.8$ & $13.05 \pm 5.0$ & $15.6 \pm 5.4$ \\
Knee $_{S}[\mathrm{deg}]$ & AP & $57.9 \pm 8.0$ & $53.5 \pm 12.1$ & $54.6 \pm 14.4$ \\
& ML & $51.9 \pm 14.4$ & $40.3 \pm 15.2$ & $42.2 \pm 14.5$ \\
Ankle $_{S}[\mathrm{deg}]$ & AP & $22.6 \pm 7.6$ & $12.2 \pm 2.4$ & $11.8 \pm 3.6$ \\
& ML & $32.1 \pm 18.4$ & $15.5 \pm 7.0$ & $17.3 \pm 6.6$ \\
\hline
\end{tabular}
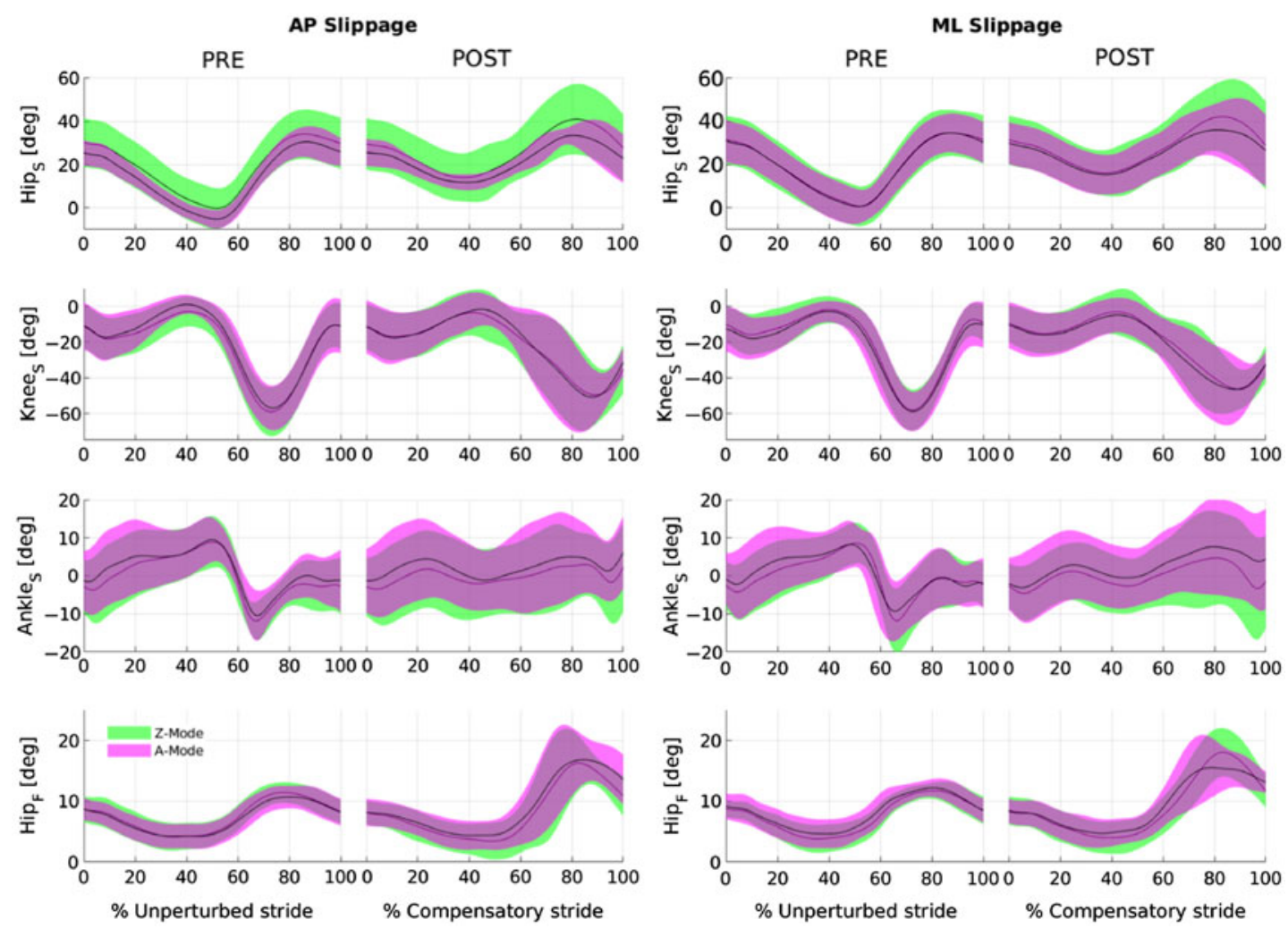

Fig. 4. Lower limb angular excursions. Joint angles (mean \pm 1 standard deviation error band) into the sagittal $\left(\mathrm{Hip}_{\mathrm{S}}, \mathrm{Knee}_{\mathrm{S}}\right.$, and Ankle $\mathrm{S}$ ) and the frontal $\left(\mathrm{Hip}_{\mathrm{F}}\right)$ planes, during anteroposterior (AP) and mediolateral (ML) slippages (left and right panels, respectively). Data before (PRE) and after (POST) the onset of the perturbation are depicted as a percentage of the unperturbed and compensatory strides, respectively, while the APO was working in Z-mode (green area) and A-mode (magenta area).

As far as the POST data are concerned, a significant main effect of the assistive strategy was observed at $\mathrm{LO}_{\mathrm{P}}$ (Table VI). Post hoc analysis revealed that $\mathrm{MoS}_{\mathrm{S}}$ was significantly higher during the Z-mode than during the A-mode (Table V). In addition, an interaction effect was reported at $\mathrm{TD}_{\mathrm{P}}$ (Table VI): during AP disturbances, the $\mathrm{MoS}_{\mathrm{S}}$ was higher for $\mathrm{Z}$-mode than for A-mode; vice versa during ML disturbances.

3.3.2. Dynamic stability in the frontal plane. The values of MoSF are reported in Table VII. The perturbation significantly affected the MoSF at TOR/LOP and HSR/TDP ( $t$-test; $p<0.0001$ and 
Table IV. Two-way repeated measures ANOVA on RoMs. $p$-values are reported for main effects of the perturbation direction $\left(p_{\mathrm{dir}}\right)$ and the exoskeleton-working modality $\left(p_{\text {exos }}\right)$, and for their interaction $\left(p_{\text {dir }}\right.$ exos $) . p<0.05$ are in bold.

\begin{tabular}{lccc}
\hline RoM & p $_{\text {dir }}$ & pexos & pdir*exos \\
\hline Hip $_{S}$ & 0.796 & $\mathbf{0 . 0 0 5}$ & 0.849 \\
Hip $_{F}$ & 0.869 & 0.287 & 0.527 \\
Knee $_{S}$ & 0.061 & 0.333 & 0.774 \\
Ankle $_{S}$ & 0.054 & 0.754 & 0.107 \\
\hline
\end{tabular}

Table V. Margin of stability in the sagittal plane $\left(\mathrm{MoS}_{\mathrm{S}}\right.$; mean \pm standard deviation, across subjects) estimated at each time event $\left(\mathrm{TO}_{\mathrm{L}} / \mathrm{LO}_{\mathrm{U}}, \mathrm{HS}_{\mathrm{L}} / \mathrm{TD}_{\mathrm{U}}, \mathrm{TO}_{\mathrm{R}} / \mathrm{LO}_{\mathrm{P}}\right.$, and $\left.\mathrm{HS}_{\mathrm{R}} / \mathrm{TD}_{\mathrm{P}}\right)$. Data are reported before (PRE) and after (POST) the perturbation onset, for each direction of the slippages (AP and ML) and for each exoskeleton-working modality (A- and Z-mode).

\begin{tabular}{|c|c|c|c|c|}
\hline \multirow[b]{2}{*}{ Time event } & \multirow[b]{2}{*}{ Perturbation direction } & \multirow[b]{2}{*}{ PRE } & \multicolumn{2}{|c|}{ POST } \\
\hline & & & A-mode & Z-mode \\
\hline \multirow[t]{2}{*}{$\mathrm{TO}_{\mathrm{L}} / \mathrm{LO}_{\mathrm{U}}[\mathrm{m}]$} & AP & $0.08 \pm 0.06$ & $0.07 \pm 0.07$ & $0.10 \pm 0.06$ \\
\hline & ML & $0.10 \pm 0.06$ & $0.11 \pm 0.05$ & $0.11 \pm 0.05$ \\
\hline \multirow[t]{2}{*}{$\mathrm{HS}_{\mathrm{L}} / \mathrm{TD}_{\mathrm{U}}[\mathrm{m}]$} & $\mathrm{AP}$ & $-0.27 \pm 0.07$ & $0.65 \pm 0.05$ & $0.69 \pm 0.12$ \\
\hline & ML & $-0.26 \pm 0.06$ & $0.65 \pm 0.08$ & $0.67 \pm 0.10$ \\
\hline \multirow{2}{*}{$\mathrm{TO}_{\mathrm{R}} / \mathrm{LO}_{\mathrm{P}}[\mathrm{m}]$} & $\mathrm{AP}$ & $0.13 \pm 0.05$ & $-0.01 \pm 0.12$ & $0.11 \pm 0.16$ \\
\hline & ML & $0.11 \pm 0.05$ & $0.05 \pm 0.10$ & $0.09 \pm 0.10$ \\
\hline \multirow[t]{2}{*}{$\mathrm{HS}_{\mathrm{R}} / \mathrm{TD}_{\mathrm{P}}[\mathrm{m}]$} & $\mathrm{AP}$ & $-0.24 \pm 0.07$ & $0.17 \pm 0.14$ & $0.24 \pm 0.13$ \\
\hline & ML & $-0.24 \pm 0.07$ & $0.18 \pm 0.10$ & $0.14 \pm 0.09$ \\
\hline
\end{tabular}

Table VI. Two-way repeated measures ANOVA on $\mathrm{MoS}_{\mathrm{S}}$ estimated at each time event after the perturbation onset $\left(\mathrm{LO}_{\mathrm{U}}, \mathrm{TD}_{\mathrm{U}}, \mathrm{LO}_{\mathrm{P}}\right.$, and $\left.\mathrm{TD}_{\mathrm{P}}\right) . p$-values are reported for main effects of the perturbation direction $\left(p_{\text {dir }}\right)$ and the exoskeleton-working modality $\left(p_{\text {exos }}\right)$, and for their interaction $\left(p_{\text {dir*exos }}\right) . p<0.05$ are in bold.

\begin{tabular}{lccc}
\hline Time event & $\boldsymbol{p}_{\text {dir }}$ & $\boldsymbol{p}_{\text {exos }}$ & $\boldsymbol{p}_{\text {dir }}$ exos \\
\hline $\mathrm{LO}_{\mathrm{U}}$ & 0.603 & 0.952 & 0.597 \\
$\mathrm{TD}_{\mathrm{U}}$ & 0.243 & 0.900 & 0.985 \\
$\mathrm{LO}_{\mathrm{P}}$ & 0.726 & $\mathbf{0 . 0 4 0}$ & 0.298 \\
$\mathrm{TD}_{\mathrm{P}}$ & 0.435 & 0.327 & $\mathbf{0 . 0 1 3}$
\end{tabular}

$p=0.002$, respectively), showing lower values before than those observed after the perturbation onset (Table VII).

$\mathrm{MoS}_{\mathrm{F}}$ was only affected by the direction of the perturbation at $\mathrm{TD}_{\mathrm{U}}$ (Table VIII) during the compensatory stride (i.e., POST data). Specifically, the post hoc analysis revealed that $\mathrm{MoS}_{\mathrm{F}}$ at $\mathrm{TD}_{\mathrm{U}}$ was higher during ML disturbances than during the AP ones (Table VII). No significant differences were observed between Z-mode and A-mode.

\section{Discussion}

The aim of this study was to investigate the effectiveness of a robot-mediated assistive strategy while promoting balance recovery after unexpected multidirectional slippages. This study was designed to generalize the outcome of our early report ${ }^{10}$ in order to gather further insights concerning the ability of our active pelvis exoskeleton to counteract the loss of balance due to multidirectional slippages. The results (Fig. 4; Tables V and VI) confirmed that our robot-mediated assistive strategy is effective in promoting stability in the sagittal plane, as assessed by the $\mathrm{MoS}_{\mathrm{S}}$. However, it is not effective at promoting stability in the frontal plane (Tables VII and VIII). 
Table VII. Margin of stability in the frontal plane $\left(\mathrm{MoS}_{\mathrm{F}}\right.$; mean \pm standard deviation, across subjects) estimated at each time event $\left(\mathrm{TO}_{\mathrm{L}} / \mathrm{LO}_{\mathrm{U}}, \mathrm{HS}_{\mathrm{L}} / \mathrm{TD}_{\mathrm{U}}, \mathrm{TO}_{\mathrm{R}} / \mathrm{LO}_{\mathrm{P}}\right.$, and $\left.\mathrm{HS}_{\mathrm{R}} / \mathrm{TD}_{\mathrm{P}}\right)$. Data are reported before (PRE) and after (POST) the perturbation onset, for each direction of the slippages (AP and ML) and for each exoskeleton-working modality (A- and Z-mode).

\begin{tabular}{lcccc}
\hline & & & \multicolumn{2}{c}{ POST } \\
\cline { 3 - 5 } Time event & Perturbation direction & PRE & A-mode & Z-mode \\
\hline $\mathrm{TO}_{\mathrm{L}} / \mathrm{LO}$ & & & & \\
& $\mathrm{AP}$ & $0.09 \pm 0.04$ & $0.10 \pm 0.01$ & $0.08 \pm 0.04$ \\
$\mathrm{HS}_{\mathrm{L}} / \mathrm{TD}_{\mathrm{U}}[\mathrm{m}]$ & $\mathrm{ML}$ & $0.09 \pm 0.04$ & $0.10 \pm 0.03$ & $0.09 \pm 0.04$ \\
& $\mathrm{AP}$ & $0.22 \pm 0.04$ & $0.24 \pm 0.05$ & $0.19 \pm 0.10$ \\
$\mathrm{TO}_{\mathrm{R}} / \mathrm{LO}$ & $\mathrm{ML}$ & $0.22 \pm 0.05$ & $0.25 \pm 0.08$ & $0.24 \pm 0.08$ \\
& $\mathrm{AP}$ & $0.13 \pm 0.03$ & $0.14 \pm 0.05$ & $0.16 \pm 0.06$ \\
$\mathrm{HS}_{\mathrm{R}} / \mathrm{TD}_{\mathrm{P}}[\mathrm{m}]$ & $\mathrm{ML}$ & $0.11 \pm 0.03$ & $0.15 \pm 0.05$ & $0.15 \pm 0.06$ \\
& $\mathrm{AP}$ & $0.25 \pm 0.03$ & $0.27 \pm 0.10$ & $0.27 \pm 0.07$ \\
& $\mathrm{ML}$ & $0.24 \pm 0.03$ & $0.26 \pm 0.07$ & $0.29 \pm 0.08$ \\
\hline
\end{tabular}

Table VIII. Two-way repeated measures ANOVA on $\mathrm{MoS}_{\mathrm{F}}$ estimated at each time event after the perturbation onset $\left(\mathrm{LO}_{\mathrm{U}}, \mathrm{TD}_{\mathrm{U}}\right.$,

$\mathrm{LO}_{\mathrm{P}}$, and $\mathrm{TD}_{\mathrm{P}}$ ). $p$-values are reported for main effects of the perturbation direction $\left(p_{\mathrm{dir}}\right)$ and the exoskeleton-working modality $\left(p_{\text {exos }}\right)$, and for their interaction $\left(p_{\text {dir*exos }}\right) . p<0.05$ are in bold.

\begin{tabular}{lccc}
\hline Time event & $\boldsymbol{p}_{\text {dir }}$ & $\boldsymbol{p}_{\text {exos }}$ & $\boldsymbol{p}_{\text {dir*exos }}$ \\
\hline $\mathrm{LO}_{\mathrm{U}}$ & 0.275 & 0.582 & 0.650 \\
$\mathrm{TD}_{\mathrm{U}}$ & $\mathbf{0 . 0 0 1}$ & 0.722 & 0.337 \\
$\mathrm{LO}_{\mathrm{P}}$ & 0.356 & 0.849 & 0.575 \\
$\mathrm{TD}_{\mathrm{P}}$ & 0.794 & 0.808 & 0.529 \\
\hline
\end{tabular}

\subsection{Effects of the direction of the perturbation on balance control}

As widely documented, ${ }^{27-34}$ an unexpected slippage significantly modifies the main kinematic and temporal features of the stride. In particular, according to our protocol, the duration of the compensatory stride decreased by about $25 \%$ (from $1.02 \pm 0.03 \mathrm{~s}$ to $0.79 \pm 0.05 \mathrm{~s}$ ) with a significant shortening of all its sub-phases (see Table I). In addition, the angular excursion at the hip joint in the frontal plane was altered by a significant increment of the RoM, mostly hip abduction, thus involving a greater width of the ipsilateral steps following the onset of the perturbation (Fig. 4; Tables III and IV). Furthermore, slippages significantly diminished the RoM in the sagittal plane only at the knee and ankle joints (Fig. 4; Tables III and IV) and affected both the time course and inter-subject variability of all joint patterns (Fig. 4). Remarkably, despite the different perturbation paradigms (i.e., AP vs. ML slippages), both timing of the compensatory stride (Tables I and II) and kinematic patterns of the PL (Tables III and IV) were not affected by the direction of the slippage.

The absence of a significant effect of the direction of the perturbation on hip joint kinematics in the frontal plane suggests that subjects elicited a vigorous use of the hip abductor-adductor muscles in order to minimize the relative movement between the upper body and the leading (i.e., perturbed) limb, apart from the direction of the perturbation. As a matter of fact, the literature agrees on the evidence that these muscle groups play an important role in managing the balance in the frontal plane ${ }^{35-40}$ In addition, the APO is provided with free rotational joints in the frontal plane, and thus it is not expected to significantly affect hip joint kinetics during the compensatory stride, unless for parasitic effects. Accordingly, early after the perturbation, the passive properties of the muscleskeletal system in the frontal plane, i.e., stiffness and inertia, seem to be similarly controlled across the perturbations in order to counteract the effects of the lack of balance.

Notably, the $\mathrm{MoS}_{\mathrm{F}}$ at the $\mathrm{TD}_{\mathrm{U}}$ after ML perturbations was greater than that assessed following AP ones (Tables VII and VIII), albeit subjects showed similar duration of the compensatory stride (Tables I and II) and kinematic of the limb being perturbed (Tables III and IV) between perturbation paradigms (i.e., AP vs. ML). Specifically, the distance between the external boundary of the base of support (i.e., the fifth metatarsal head of the right foot) and the extrapolated $\mathrm{CoM}(\mathrm{XCoM})$ during 
the first half of the compensatory stride was significantly greater after ML slippages than for AP ones. This experimental evidence confirms that the pendulum like dynamics of the muscle-skeletal system in the frontal plane reflects the direction of the slippage, thus requiring a context-specific counteracting action to promote balance recovery.

All in all, the different paradigms of slippage (i.e., AP vs. ML) involved different behaviors of the participants, only affecting the $\mathrm{MoS}_{\mathrm{F}}$. On the other hand, we unexpectedly observed that the hip joint angle in the frontal plane did not reflect such perturbations. Accordingly, we believe that although the APO could partially restrain the hip movement of the users in the frontal plane, balance control is mostly mediated by subjects' abd/adduction muscle groups leading them to adopt a contextdependent counteractive strategy to manage the lack of balance.

\subsection{Effects of the robot-mediated assistance on balance control}

The results confirm that the assistive strategy tested in this study modified the behavioral features of the compensatory stride mainly in terms of the kinematics at the hip joint (Fig. 4; Tables III and IV) and stability in the sagittal plane (i.e., $\mathrm{MoS}_{\mathrm{S}}$; Tables V and VI). In addition, with respect to the stability in the sagittal plane, we observed a significant interaction between the perturbation direction (i.e., AP vs. ML) and the exoskeleton working modality (i.e., A- vs. Z-mode).

More in detail, the results reveal that the robot-mediated assistance (i.e., A-mode) significantly modified the hip RoM in the sagittal plane $\left(\mathrm{RoM}_{\mathrm{Hs}}\right.$ ), reducing the flexion of the PL (Fig. 4; Tables III and IV). However, neither the hip RoM in the frontal plane nor the RoM of the knee and ankle in the sagittal plane was affected by the proposed assistive strategy. As far as stability is concerned, the robot-mediated assistance (A-mode) involved a lower $\mathrm{MoS}_{\mathrm{S}}$ computed at $\mathrm{LO}_{\mathrm{P}}$ than that assessed in transparent mode (Z-mode; Tables V and VI).

Results pertaining the effects of the robot-mediated assistive strategy on hip joint kinematics confirm our previous findings, ${ }^{10}$ revealing that the proposed approach promotes the lowering of the perturbed foot by mostly reducing hip flexion in the late swing phase (Fig. 4). Concerning the MoS, in this study we obtained different results compared to our previous report. In particular, here we observed that the $\mathrm{MoS}_{\mathrm{S}}$ in A-mode, averaged between AP and ML conditions and computed at the $\mathrm{LO}_{\mathrm{P}}$, was about $0.02 \pm 0.11 \mathrm{~m}$, while the homologous one in Z-mode was about $0.10 \pm 0.13 \mathrm{~m}$, documenting a backward shift of the XCoM when the exoskeleton worked in transparent mode. A similar behavior can be observed if separately looking at AP and ML perturbations.

The greater backward shift of the XCoM observed at the beginning of the contralateral step after the onset of the perturbation (i.e., $\mathrm{LO}_{\mathrm{P}}$ ) in $\mathrm{Z}$-mode actually reveals that the subjects were experiencing a stronger backward loss of balance than in A-mode. As a matter of fact, a greater MoS at the $\mathrm{LO}_{\mathrm{P}}$, as observed for Z-mode, mostly suggests that the $\mathrm{XCoM}$ is more closely projected toward the posterior boundary of the base of support. Hence, in Z-mode, the impulse needed to unbalance a subject toward a backward loss of balance is supposed to be smaller than that required in A-mode, thus reflecting a more critical condition of the former one. Differently, in A-mode, the position of the XCoM is almost coinciding with the projection of the CoM along the AP axis, thus suggesting that the subject likely resembled an almost vertical pendulum with smaller CoM velocity, compared to the Z-mode. Therefore, according to this evidence, it is possible to infer that, thanks to the robot-mediated strategy, users could recover their balance by minimizing the number of compensative steps.

It is worth noting that the post hoc analysis on $\mathrm{MoS}_{\mathrm{S}}$ at the $\mathrm{TD}_{\mathrm{P}}$ showed an interaction between perturbation direction (AP vs. ML) and exoskeleton working mode (A- vs Z-modes). In particular, although the XCoM always dropped within the base of support at the end of the compensatory stride (i.e., $\mathrm{MoS}_{\mathrm{S}}$ is always positive at $\mathrm{TD}_{\mathrm{P}}$ compared to homologous values pre-perturbation), the $\mathrm{MoS}_{\mathrm{S}}$ was higher for AP perturbations when the APO was working in Z-mode, compared to Amode. A different trend was observed for the ML perturbations, albeit the difference between the exoskeleton working modes was smaller than the previous ones. Furthermore, the $\mathrm{MoS}_{\mathrm{S}}$ did not change between perturbation directions when the APO was working in A-mode (Tables V and VI). This result suggests, on one hand, that the users can adopt a context-specific (i.e., perturbation-based) reactive behavior when the exoskeleton works in transparent mode (Z-mode). On the other hand, the assistive contribution of the exoskeleton forces the users to accomplish similar dynamical stability in the sagittal plane regardless the direction of the perturbation. The dynamics after the perturbation can hence be significantly influenced by the assistive contribution of the exoskeleton, thus confirming that if the exoskeleton is properly tuned, it can effectively promote balance recovery after a slippage. ${ }^{10}$ 
We also observed an interaction effect between the direction of the slippages (AP vs. ML) and the APO working modalities (Z- vs. A-modes) in the duration of the second double support phase of the compensatory stride ( $\mathrm{DS}_{2}$; Tables I and II). This is consistent with the evidence that the A-mode is enabled after about $0.35-0.40 \mathrm{~s}$ from the onset of the perturbation ${ }^{10-17}$ and suggests that users adopted different strategies to manage AP and ML slippages while being assisted by the APO: in the former case (AP perturbations), subjects adopted a shorter $\mathrm{DS}_{2}$, thus accelerating the shift from the perturbed to the UL; in the latter case (ML perturbations), the assistive strategy slightly increased the double support phase, thus leading the users to take advantage of a longer bipedal configuration to control the balance in the frontal plane.

Another significant result concerns the effects of the assistive strategy on the balance control in the frontal plane $\left(\mathrm{MoS}_{\mathrm{F}}\right.$; Table VII). Specifically, our analysis revealed that our robot-mediated assistance never modifies the $\mathrm{MoS}_{\mathrm{F}}$. This is in accordance with the fact that the adopted APO cannot actively drive hip abd-adduction, so that its assistance is mainly expected to promote stability in the sagittal plane.

To the best of our knowledge, a novel promising concept of a wearable hip exoskeleton, aimed at counteracting balance loss, has been recently introduced in the literature. ${ }^{41}$ This device was designed to maintain balance in both the sagittal and frontal planes by using powered actuation for hip flexextension and abd-adduction, relying on a novel control strategy based on the XCoM. Unfortunately, in this study, the results concerning balance stability against perturbations appear very preliminary, since they refer to a single young adult while walking at very slow speeds $(0.45-0.65 \mathrm{~m} / \mathrm{s})$, and managing pushes delivered manually by an experimenter. Therefore, we are unable to compare the performance of our robot-mediated strategy with the current literature. In this respect, due to the expected forthcoming pervasiveness of such wearable robotic platforms in our society, further research is strongly required in order to set benchmark criteria to analyze and compare different platforms.

\section{Conclusion}

Wearable robotic platforms are becoming more and more widespread in our society for both rehabilitative and assistive purposes. One of the main open challenges pertains to the ability of these platforms to promote balance recovery after unexpected disturbances during the activities of daily living. For this reason, we tested the effectiveness of our robot-mediated strategy while older adults were asked to manage multidirectional slippages. The results revealed that our approach allows the users to better control their balance in the sagittal plane only (i.e., $\mathrm{MoS}_{\mathrm{S}}$ ), for both AP and ML slippages. The balance control in the frontal plane is not yet effectively addressed by our system due to some intrinsic limitations of our platform, such as the absence of any active control of the hip abd-adduction.

\section{Acknowledgments}

This work was supported by the EU Commission through the FP7 project CYBERLEGs (The CYBERnetic LowEr-Limb CoGnitive Orthoprosthesis, Grant Agreement no. 287894) and the H2020 project CYBERLEGs Plus Plus (The CYBERnetic LowEr-Limb CoGnitive Ortho-prosthesis Plus Plus, Grant Agreement no. 731931), and the institutional funds from The BioRobotics Institute, Scuola Superiore Sant'Anna.

\section{Competing Interests}

Nicola Vitiello and Silvestro Micera are the co-founders of and have financial interests in the company IUVO SRL, a spin-off company of Scuola Superiore Sant'Anna aiming at commercially exploiting the APO technology. Nicola Vitiello is the co-inventor of two patent applications protecting the APO technology.

\section{References}

1. T. Masud and R. O. Morris, "Epidemiology of falls," Age Ageing. 30, 3-7 (2001). doi: 10.1093/ ageing/30.1.3.

2. L. Z. Rubenstein, "Falls in older people: Epidemiology, risk factors and strategies for prevention," Age Ageing. 35(Suppl 2), ii37-ii41 (2006). doi: 10.1093/ageing/afl084. 
3. A. F. Ambrose, G. Paul and J. M. Hausdorff, "Risk factors for falls among older adults: A review of the literature," Maturitas. 75, 51-61 (2013). doi: 10.1016/j.maturitas.2013.02.009.

4. R. D. Laird, S. Studenski, S. Perera and D. Wallace, "Fall history is an independent predictor of adverse health outcomes and utilization in the elderly," Am. J. Manag. Care. 7, 1133-1138 (2001).

5. L. Yardley and H. Smith, "A prospective study of the relationship between feared consequences of falling and avoidance of activity in community-living older people," Gerontologist 42, 17-23 (2002). doi: $10.1093 /$ geront/42.1.17.

6. R. Rajagopalan, I. Litvan and T.-P. Jung, "Fall prediction and prevention systems: Recent trends, challenges, and future research directions," Sensors 17, 2509 (2017). doi: 10.3390/s17112509.

7. M. D. Grabiner, M. Lou Bareither, S. Gatts, J. Marone and K. L. Troy, "Task-specific training reduces trip-related fall risk in women," Med. Sci. Sports. Exerc. 44, 2410-2414 (2012). doi: 10.1249/MSS.0b013e318268c89f.

8. K. Hauer, C. Becker, U. Lindemann and N. Beyer, "Effectiveness of physical training on motor performance and fall prevention in cognitively impaired older persons: A systematic review," Am. J. Phys. Med. Rehabil. 85, 847-57 (2006). doi: 10.1097/01.phm.0000228539.99682.32.

9. L. A. Perula, F. Varas-Fabra, V. Rodriguez, R. Ruiz-Moral, J. A. Fernandez, J. Gonzalez, C. J. Perula, A. M. Roldan, C. de Dios and E. S. C. Group, "Effectiveness of a multifactorial intervention program to reduce falls incidence among community-living older adults: A randomized controlled trial," Arch. Phys. Med. Rehabil. 93, 1677-1684 (2012). doi: 10.1016/j.apmr.2012.03.035.

10. V. Monaco, P. Tropea, F. Aprigliano, D. Martelli, A. Parri, M. Cortese, R. Molino-Lova, N. Vitiello and S. Micera, "An ecologically-controlled exoskeleton can improve balance recovery after slippage," Sci. Rep. 7, 46721 (2017). doi: 10.1038/srep46721.

11. A. D. Kuo, "Stabilization of lateral motion in passive dynamic walking," Int. J. Rob. Res. 18, 917-930 (1999). doi: 10.1177/02783649922066655.

12. D. Martelli, V. Monaco, L. Bassi Luciani and S. Micera, "Angular momentum during unexpected multidirectional perturbations delivered while walking," IEEE Trans. Biomed. Eng. 60(7), 1785-1795 (2013). doi: 10.1109/TBME.2013.2241434.

13. L. Bassi Luciani, V. Genovese, V. Monaco, L. Odetti, E. Cattin and S. Micera, "Design and Evaluation of a new mechatronic platform for assessment and prevention of fall risks," J. Neuroeng. Rehabil.9, 51 (2012). doi: 10.1186/1743-0003-9-51.

14. F. Aprigliano, D. Martelli, S. Micera and V. Monaco, "Intersegmental coordination elicited by unexpected multidirectional slipping-like perturbations resembles that adopted during steady locomotion," $J$. Neurophysiol. 115, 728-740 (2016). doi: 10.1152/jn.00327.2015.

15. F. Giovacchini, F. Vannetti, M. Fantozzi, M. Cempini, M. Cortese, A. Parri, T. Yan, D. Lefeber and N. Vitiello, "A light-weight active orthosis for hip movement assistance," Rob. Auton. Syst. 73, 123-134 (2015). doi: 10.1016/j.robot.2014.08.015.

16. D. Martelli, F. Vannetti, M. Cortese, P. Tropea, F. Giovacchini, S. Micera, V. Monaco and N. Vitiello, "The effects on biomechanics of walking and balance recovery in a novel pelvis exoskeleton during zero-torque control," Robotica. 32, 1317-1330 (2014). doi: 10.1017/S0263574714001568.

17. P. Tropea, N. Vitiello, D. Martelli, F. Aprigliano, S. Micera and V. Monaco, "Detecting slipping-like perturbations by using adaptive oscillators," Ann. Biomed. Eng. 43, 416-426 (2015). doi: 10.1007/s10439-014-1175-5.

18. F. Aprigliano, V. Monaco, P. Tropea, D. Martelli, N. Vitiello and S. Micera, "Effectiveness of Assistive Torque Patterns Supplied by a Pelvis Exoskeleton After Slippages: A Pilot Study." Proceedings of the 4th International Conference on NeuroRehabilitation (ICNR2018), October 16-20, 2018, Pisa, Italy (2019) pp. 273-277. doi: 10.1007/978-3-030-01845-0_55.

19. A. L. Bell, D. R. Pedersen and R. A. Brand, "A comparison of the accuracy of several hip center location prediction methods," J. Biomech. 23, 617-621 (1990). doi: 10.1016/0021-9290(90)90054-7.

20. R. B. Davis, S. Õunpuu, D. Tyburski and J. R. Gage, "A gait analysis data collection and reduction technique," Hum. Mov. Sci. 10, 575-587 (1991). doi: 10.1016/0167-9457(91)90046-Z.

21. D. A. Winter, "Biomechanics and motor control of human Gait," Biomech. Mot. Control Hum. Gait Norm. Elder. Pathol. 2, 143 (1991).

22. F. Yang and Y. C. Pai, "Can sacral marker approximate center of mass during gait and slip-fall recovery among community-dwelling older adults?," J. Biomech. 47, 3807-3812 (2014). doi: 10.1016/j.jbiomech.2014.10.027.

23. V. Monaco and S. Micera, "Age-related neuromuscular adaptation does not affect the mechanical efficiency of lower limbs during walking," Gait Posture. 36, 350-355 (2012). doi: 10.1016/j.gaitpost.2012.03.031.

24. L. Quagliarella, N. Sasanelli and V. Monaco, "Drift in posturography systems equipped with a piezoelectric force platform: Analysis and numerical compensation," IEEE Trans. Instrum. Meas. 57, 997-1004 (2008). doi: 10.1109/TIM.2007.913833.

25. A. L. Hof, M. G. J. Gazendam and W. E. Sinke, "The condition for dynamic stability," J. Biomech. 38, 1-8 (2005). doi: 10.1016/j.jbiomech.2004.03.025.

26. A. L. Hof, "The 'extrapolated center of mass' concept suggests a simple control of balance in walking," Hum. Mov. Sci. 27, 112-125 (2008). doi: 10.1016/j.humov.2007.08.003.

27. F. Aprigliano, D. Martelli, P. Tropea, G. Pasquini, S. Micera and V. Monaco, "Aging does not affect the intralimb coordination elicited by slip-like perturbation of different intensities," J. Neurophysiol. 118(3), 1739-1748 (2017). doi: 10.1152/jn.00844.2016. 
28. T. E. Lockhart, J. C. Woldstad and J. L. Smith, "Effects of age-related gait changes on the biomechanics of slips and falls," Ergonomics. 46, 1136-1160 (2003). doi: 10.1080/0014013031000139491.

29. D. Martelli, F. Aprigliano, P. Tropea, G. Pasquini, S. Micera and V. Monaco, "Stability against backward balance loss: Age-related modifications following slip-like perturbations of multiple amplitudes," Gait Posture. 53, 207-214 (2017). doi: 10.1016/j.gaitpost.2017.02.002.

30. R. Cham and M. S. Redfern, "Lower extremity corrective reactions to slip events," J. Biomech. 34, 14391445 (2001). doi: 10.1016/S0021-9290(01)00116-6.

31. M. S. Redfern, R. Cham, K. Gielo-Perczak, R. Grönqvist, M. Hirvonen, H. Lanshammar, M. Marpet, C. Y. C. Pai and C. Powers, "Biomechanics of slips," Ergonomics. 44(13), 1138-1166 (2001). doi: 10.1080/00140130110085547.

32. F. Aprigliano, D. Martelli, P. Tropea, S. Micera and V. Monaco, "Effects of slipping-like perturbation intensity on the dynamical stability," Conf. Proc. IEEE Eng. Med. Biol. Soc. 2015, 5295-5298 (2015). doi: 10.1109/EMBC.2015.7319586.

33. R. Ferber, L. R. Osternig, M. H. Woollacott, N. J. Wasielewski and J. H. Lee, "Reactive balance adjustments to unexpected perturbations during human walking," Gait Posture. 16, 238-248 (2002). doi: 10.1016/S0966-6362(02)00010-3.

34. P. Tropea, D. Martelli, F. Aprigliano, S. Micera and V. Monaco, "Effects of aging and perturbation intensities on temporal parameters during slipping-like perturbations," Conf. Proc. IEEE Eng. Med. Biol. Soc. 2015, 5291-5294 (2015). doi: 10.1109/EMBC.2015.7319585.

35. D. A. Winter, "Human balance and posture control during standing and walking," Gait Posture. 3, 193-214 (1995). doi: 10.1016/0966-6362(96)82849-9.

36. M. Inacio, A. S. Ryan, W. N. Bair, M. Prettyman, B. A. Beamer and M. W. Rogers, "Gluteal muscle composition differentiates fallers from non-fallers in community dwelling older adults," BMC Geriatrics. 14, 37 (2014). doi: 10.1186/1471-2318-14-37.

37. M. L. Mille, M. E. Johnson, K. M. Martinez and M. W. Rogers, "Age-dependent differences in lateral balance recovery through protective stepping," Clin. Biomech. 20, 607-616 (2005). doi: 10.1016/j.clinbiomech.2005.03.004.

38. L. Dipietro, "Physical activity in aging: Changes in patterns and their relationship to health and function," J. Gerontol. A. Biol. Sci. Med. Sci. 56, 13-22 (2001). doi: 10.1093/gerona/56.suppl_2.13.

39. J. S. Gottschall, N. Okita and R. C. Sheehan, "Muscle activity patterns of the tensor fascia latae and adductor longus for ramp and stair walking," J. Electromyogr. Kinesiol. 22, 67-73 (2012). doi: 10.1016/j.jelekin.2011.10.003.

40. M. W. Rogers and M.-L. Mille, "Lateral stability and falls in older people," Exerc. Sport Sci. Rev. 31, 182-187 (2003). doi: 10.1097/00003677-200310000-00005.

41. T. Zhang, M. Tran and H. Huang, "Design and experimental verification of hip exoskeleton with balance capacities for walking assistance," IEEE ASME Trans. Mechatron. 23, 274-285 (2018). doi: 10.1109/TMECH.2018.2790358. 